\title{
Chiral and deconfinement transitions in a magnetic background using the functional renormalization group with the Polyakov loop
}

\author{
Jens O. Andersen, ${ }^{a}$ William R. Naylor ${ }^{a}$ and Anders Tranberg $^{b}$ \\ ${ }^{a}$ Department of Physics, Norwegian University of Science and Technology, \\ Høgskoleringen 5, N-7491 Trondheim, Norway \\ ${ }^{b}$ Faculty of Science and Technology, University of Stavanger, \\ N-4036 Stavanger, Norway \\ E-mail: andersen@tf.phys.ntnu.no, william.naylor@ntnu.no, \\ anders.tranberg@uis.no
}

ABStract: We use the Polyakov loop coupled quark-meson model to approximate low energy QCD and present results for the chiral and deconfinement transitions in the presence of a constant magnetic background $B$ at finite temperature $T$ and baryon chemical potential $\mu_{B}$. We investigate effects of various gluonic potentials on the deconfinement transition with and without a fermionic backreaction at finite $B$. Additionally we investigate the effect of the Polyakov loop on the chiral phase transition, finding that magnetic catalysis at low $\mu_{B}$ is present, but weakened by the Polyakov loop.

KeYwords: Thermal Field Theory, Renormalization Group, Phase Diagram of QCD

ArXiv ePrint: 1311.2093 


\section{Contents}

1 Introduction 1

2 Quark-meson model and the functional renormalization group 2

3 Adding the Polyakov loop 4

$\begin{array}{lll}4 & \text { Numerical implementation and the glue potential } & 7\end{array}$

$\begin{array}{lll}5 & \text { Results at finite magnetic field } & 10\end{array}$

$\begin{array}{llr}6 & \text { Summary and outlook } & 14\end{array}$

\section{Introduction}

Knowledge of the equation of state and the phase diagram of QCD is essential in understanding the properties of the deconfined matter created in heavy-ion collisions as well as the properties of compact stars and their quark cores. In non-central heavy-ion collisions, large time-dependent magnetic fields are generated during the experiment [1-3]. The maximum strength of these magnetic fields is on the order of $10^{19}$ Gauss $\left(q B \sim 6 m_{\pi}^{2}\right)$. Likewise, very strong magnetic fields exist inside magnetars [4]. These may be several orders of magnitude larger than the magnetic fields in ordinary neutron stars. On the surface, the magnetic field may be as strong as $10^{14}-10^{15}$ Gauss and it could be as strong as $10^{16}-10^{19}$ Gauss in the interior of the star. This has spurred the interest in strongly interacting matter at finite temperature, density and magnetic field, see for example ref. [5] for a recent review.

The phase boundary in $\left(T, \mu_{B}, B\right)$ space is therefore of great interest; however due to the infamous sign problem, one cannot use the standard techniques of lattice calculations at finite $\mu_{B}$. At zero $\mu_{B}$ and finite $B$, there is no sign problem and so one can calculate the phase diagram in the $T, B$ plane using Monte-Carlo methods. Recent lattice calculations $[6,7]$ suggest that for physical quark masses, the transition temperature for the chiral transition is a decreasing function of the magnetic field $B$, while for larger values of the quark masses corresponding to $m_{\pi} \simeq 400 \mathrm{MeV}$, the temperature is an increasing function of $B[8,9]$. The qualitative behavior of the transition temperature for physical quark masses is in disagreement with model calculations using either the (Polyakov-loop extended) Nambu-Jona-Lasinio ((P)NJL) model or the (Polyakov-loop extended) quarkmeson model $((\mathrm{P}) \mathrm{QM})$; in these models, the critical temperature is an increasing function of the magnetic field, see e.g. [10-20]. Possible resolutions to the disagreement have been suggested [21-28] and we will discuss these at the end of the paper. 
In a previous paper [17], two of us used the two-flavor three-color quark-meson model and the functional renormalization group [29] to map out the phase diagram in the $\mu_{B}-T$ plane for different values of the magnetic field (see also refs. [30, 31]). In the present paper, we add the Polyakov loop to the model to include certain aspects of confinement $[32,33]$. In particular, we investigate a set of possible implementations of the Polyakov loop and how they effect both the chiral and deconfinement transitions. In the context of the functional renormalization group, this was studied in ref. [16] at zero baryon chemical potential.

The paper is organized as follows: in section 2 we briefly discuss the functional renormalization group implementation of the quark-meson model in a constant magnetic background. In section 3 we add the Polyakov loop variable to the model and review the three gluonic potentials we have used in this work. Section 4 explains the numerical implementation and the effects of the various gluonic potentials. In section 5 we discuss our results for the deconfinement and chiral transitions. Finally, in section 6 , we summarise the main results and comment on the disagreement between lattice and model calculations at finite $B$ and $\mu_{B}=0$.

\section{Quark-meson model and the functional renormalization group}

The quark meson model is the linear sigma model coupled to two massless quark flavors via a Yukawa coupling. The $O(4)$-invariant Euclidean Lagrangian for the model is

$$
\begin{aligned}
\mathcal{L}= & \bar{\psi}\left[\gamma_{\mu} D_{\mu}-\mu \gamma_{4}+g\left(\sigma-i \gamma_{5} \boldsymbol{\tau} \cdot \boldsymbol{\pi}\right)\right] \psi+\frac{1}{2}\left[\left(\partial_{\mu} \sigma\right)^{2}+\left(\partial_{\mu} \boldsymbol{\pi}\right)^{2}\right] \\
& +\frac{1}{2} m^{2}\left[\sigma^{2}+\boldsymbol{\pi}^{2}\right]+\frac{\lambda}{4}\left[\sigma^{2}+\boldsymbol{\pi}^{2}\right]^{2}-h \sigma
\end{aligned}
$$

where $\sigma$ is the sigma field, $\boldsymbol{\pi}$ denotes the pions, $\left(\pi_{1}, \pi_{2}, \pi_{3} \equiv \pi_{0}\right)$ and $\boldsymbol{\tau}$ are the Pauli matrices, $\mu=\operatorname{diag}\left(\mu_{u}, \mu_{d}\right)$ is the quark chemical potential, where $\mu_{u}$ and $\mu_{d}$ are the chemical potential for the $u$ and $d$ quarks, respectively. We set $\mu_{u}=\mu_{d}$ so that we are working at zero isospin chemical potential, $\mu_{I}=\frac{1}{2}\left(\mu_{u}-\mu_{d}\right)=0$. The baryon chemical potential is given by $\mu_{B}=3 \mu$. When we couple the quark-meson model to an Abelian gauge field, we replace the partial derivatives by the covariant ones for the charged quarks as well as for the charged pions. The covariant derivative $D_{\mu}$ couples to the charged fields, $D_{\mu}=\partial_{\mu}-i q A_{\mu}^{\mathrm{EM}}$, where $q$ is the charge of the field, $2 / 3 e,-1 / 3 e$ and $\pm e$ for the up quark, down quark, and the charged pions respectively. With the addition of the Polyakov loop, given in section 3, a coupling between the quarks and a constant background gauge field is added to the covariant derivative. The Euclidean $\gamma$ matrices are given by $\gamma_{j}=i \gamma_{M}^{j}$, $\gamma_{4}=\gamma_{M}^{0}$, and $\gamma_{5}=-\gamma_{M}^{5}$, where the index $M$ denotes Minkowski space. The fermion field is an isospin doublet,

$$
\psi=\left(\begin{array}{l}
u \\
d
\end{array}\right) .
$$

The coupling to the Maxwell field reduces the $O(4)$ symmetry, or equally, $\mathrm{SU}(2)_{V} \times \mathrm{SU}(2)_{A}$ to $\mathrm{U}(1) \times \mathrm{U}(1)_{A}$ simply because the electric charges of the $u$ and $d$ quarks are different. The first term is a symmetry that corresponds to a rotation of the $u$ and $d$ fields with 
opposite phase, $u \rightarrow u e^{i \delta}$ and $d \rightarrow d e^{-i \delta}$. The complex field $\Delta \equiv \frac{1}{\sqrt{2}}\left(\pi_{1}+i \pi_{2}\right) \equiv \pi^{+}$is simultaneouesly rotated $\Delta \rightarrow \Delta e^{2 i \delta}$. The second is a chiral rotation of the $u$ and $d$ fields with opposite phase, $u \rightarrow e^{i \gamma_{5} \delta} u$ and $d \rightarrow e^{-i \gamma_{5} \delta} d$. The complex field $v \equiv \frac{1}{\sqrt{2}}\left(\sigma+i \gamma_{5} \pi_{0}\right)$ is simultaneously rotated $v \rightarrow e^{2 i \gamma_{5} \delta} v$. The $O(4)$ invariant $\sigma^{2}+\pi_{0}^{2}+\pi_{1}^{2}+\pi_{2}^{2}$ now splits into the two $O(2)$ invariants $v^{\dagger} v$ and $\pi^{+} \pi^{-}$, where $\pi^{-}=\Delta^{\dagger} / \sqrt{2}$. In this case, we have in principle two Yukawa couplings, two mass terms and three different coupling constants.

If $h \neq 0$, the $\mathrm{U}(1)_{A}$ symmetry is explicitly broken, otherwise it is spontaneously broken in the vacuum. Either way, the symmetry is reduced. If the symmetry is broken spontaneously, the $\pi_{0}$ is a Goldstone boson, while if the symmetry is broken explicitly it is a pseudo-Goldstone boson. The charged bosons are no longer (pseudo)Goldstone bosons. The $\mathrm{U}(1)_{A}$ symmetry is broken in the vacuum by a nonzero expectation value $\phi$ for the sigma field and we make the replacement

$$
\sigma \rightarrow \phi+\tilde{\sigma}
$$

where $\tilde{\sigma}$ is a quantum fluctuating field. The tree-level potential then becomes

$$
U_{\Lambda}=\frac{1}{2} m_{\Lambda}^{2} \phi^{2}+\frac{\lambda_{\Lambda}}{4} \phi^{4}-h \phi .
$$

Note that we have introduced a subscript $\Lambda$ on $U, m^{2}$, and $\lambda$, where $\Lambda$ is the ultraviolet cutoff of the theory. This is a reminder that these are unrenormalized quantities. ${ }^{1}$

We will follow Wetterich's implementation of the renormalization group ideas based on the effective average action $\Gamma_{k}[\varphi][29]$. This action is a functional of a set of background fields that are denoted by $\varphi . \Gamma_{k}[\varphi]$ satisfies an integro-differential flow equation in the variable $k$, to be specified below. The subscript $k$ indicates that all the modes $p$ between the ultraviolet cutoff $\Lambda$ of the theory and $k$ have been integrated out. When $k=\Lambda$ no modes have been integrated out and $\Gamma_{\Lambda}$ equals the classical action $S$. On the other hand, when $k=0$, all the momentum modes have been integrated out and $\Gamma_{0}$ equals the full quantum effective action. The flow equation then describes the flow in the space of effective actions as a function of $k$.

In order to implement the renormalization group ideas, one introduces a regulator function $R_{k}(p)$. The function $R_{k}(p)$ is large for $p<k$ and small for $p>k$ whenever $0<k<\Lambda$, and $R_{\Lambda}(p)=\infty$. These properties ensure that the modes below $k$ are heavy and decouple, and only the modes between $k$ and the UV cutoff $\Lambda$ are light and integrated out. The choice of regulator function has been discussed in detail in the literature and some choices are better than others due both to their analytical and stability properties, see for example [34].

The flow equation for the effective action cannot be solved exactly so one must make tractable and yet physically sound approximations. The first approximation in a derivative expansion is the local-potential approximation (LPA) where the flow equation for $\Gamma_{k}$ reduces to a flow equation for an effective potential $U_{k}(\phi)$. In this case one sets the wave-function renormalization factors equal to one. ${ }^{2}$ Going beyond the local-potential

\footnotetext{
${ }^{1}$ The symmetry breaking term is equivalent to an external field that does not flow and therefore $h=h_{\Lambda}$.

${ }^{2}$ Higher-order derivative operators that are consistent with the symmetries are also neglected in the LPA.
} 
approximation, one would have to solve a set of coupled equations for the wave-function renormalization factors as done in the recent paper by Kamikado and Kanazawa [19]. ${ }^{3}$ Moreover, since the $\mathrm{SU}(2)_{A}$ symmetry is broken by the magnetic field, as explained above, the effective potential is therefore a function of these two invariants. This is similar to the case of two-color QCD with a baryon chemical potential [35] or three-color QCD with an isospin chemical potential [36]. In the LPA, the effective action then takes the form

$$
\Gamma_{k}[|v|,|\Delta|]=\int_{0}^{\beta} d \tau \int d^{3} x\left\{\frac{1}{2}\left[\left(\partial_{0} \sigma\right)^{2}+\left(\partial_{0} \pi^{2}\right)\right]+\frac{1}{2}\left[(\nabla \sigma)^{2}+\left(\nabla \pi^{2}\right)\right]+U_{k}(|v|,|\Delta|)\right\} .
$$

However, since we do not have a charged pion condensate, the effective potential can be evaluated at $\pi^{+} \pi^{-} \equiv 0$, but the flow equation still depends on both partial derivatives, $\frac{\partial U_{k}}{\partial|v|}$ and $\frac{\partial U_{k}}{\partial|\Delta|}$, of the potential $U_{k}$ in field space. At the mean-field level these derivatives are identical, beyond they are not. In order to make the problem numerically tractable, we make the approximation that they are equal. With these approximations and defining $\rho=|v|$, the flow equation for the potential $U_{k}[\rho, 0]$ then reads $[16,17]$

$$
\begin{aligned}
\partial_{k} U_{k}[\rho, 0]= & \frac{k^{4}}{12 \pi^{2}}\left\{\frac{1}{\omega_{1, k}}\left[1+2 n_{B}\left(\omega_{1, k}\right)\right]+\frac{1}{\omega_{k, 2}}\left[1+2 n_{B}\left(\omega_{2, k}\right)\right]\right\} \\
& +k \frac{|q B|}{2 \pi^{2}} \sum_{m=0}^{\infty} \frac{1}{\omega_{1, k}} \sqrt{k^{2}-p_{\perp}^{2}(q, m, 0)} \theta\left(k^{2}-p_{\perp}^{2}(q, m, 0)\right)\left[1+2 n_{B}\left(\omega_{1, k}\right)\right] \\
& -\frac{N_{c}}{2 \pi^{2}} k \sum_{s, f, m=0}^{\infty} \frac{\left|q_{f} B\right|}{\omega_{q, k}} \sqrt{k^{2}-p_{\perp}^{2}\left(q_{f}, m, s\right)} \theta\left(k^{2}-p_{\perp}^{2}\left(q_{f}, m, s\right)\right)\left[1-n_{F}^{+}\left(\omega_{q, k}\right)-n_{F}^{-}\left(\omega_{q, k}\right)\right],
\end{aligned}
$$

where we have defined $\omega_{1, k}=\sqrt{k^{2}+U_{k}^{\prime}}, \omega_{2, k}=\sqrt{k^{2}+U^{\prime}+2 U_{k}^{\prime \prime} \rho}, \omega_{q, k}=\sqrt{k^{2}+2 g^{2} \rho}$, $p_{\perp}^{2}(q, m, s)=(2 m+1-s)|q B|, n_{B}(x)=1 /\left(e^{\beta x}-1\right), \rho=\frac{1}{2} \phi^{2}$ and $n_{F}^{ \pm}(x)=1 /\left(e^{\beta(x \pm \mu)}+1\right)$, however the fermionic distribution functions will be transformed to eqs. (3.3) and (3.4) when we add the Polyakov loop.

At zero temperature, the Bose distribution function vanishes and the Fermi distribution function becomes a step function. Furthermore, if we set $\mu=0$, this step function vanishes and we obtain the flow equation in the vacuum.

\section{Adding the Polyakov loop}

The Polyakov loop $\Phi$ is given by the thermal expectation value of the trace of the Wilson line, i.e.

$$
\Phi=\frac{1}{N_{c}}\left\langle\operatorname{Tr}_{c} L\right\rangle,
$$

where the trace is in color space and

$$
L=\mathcal{P} \exp \left[i \int_{0}^{\beta} d \tau A_{4}\right],
$$

\footnotetext{
${ }^{3}$ In this work, since a magnetic field breaks rotational symmetry, one must use two different wavefunction renormalization factors $Z_{k}^{\|}$and $Z_{k}^{\perp}$, where $\|$ and $\perp$ are the parallel and perpendicular directions relative to the magnetic field.
} 
where $A_{4}=i A_{0}$ and $A_{0}=\delta_{\mu 0} \mathcal{A}_{a}^{\mu} t^{a}$. Here $\mathcal{A}_{a}^{\mu}$ are the $\mathrm{SU}(3)_{c}$ gauge fields and the generators are $t^{a}=\frac{1}{2} \lambda^{a}$, where $\lambda^{a}$ are the Gell-Mann matricies. The Wilson line is a complex variable and so $\Phi$ is not equal to $\bar{\Phi}=\frac{1}{N_{c}}\left\langle\operatorname{Tr}_{c} L^{\dagger}\right\rangle$ in general. It is known that $\Phi=\bar{\Phi}$ at mean field level, but in the present work this is only true at zero baryon chemical potential. The Polyakov loop is an order parameter for deconfinement in pure-glue QCD. Under the center symmetry $Z_{N}$, it transforms as $\Phi \rightarrow e^{2 \pi i n / N_{c}}$, where $n=0,1,2 \ldots, N_{c}-1$. At low temperatures, i.e. in the confined phase, we have $\Phi \approx 0$, while in the deconfined phase we have $\Phi \approx 1$.

Coupling the Polyakov loop to the QM model gives a more physically accurate model of the quark sector and allows us to explore both the chiral and deconfinement transitions of low energy QCD. This is done by introducing a constant background temporal gauge field $\delta_{\mu 0} \mathcal{A}_{a}^{\mu}$ via the covariant derivative for the quarks $D_{\mu} \rightarrow D_{\mu}-i \delta_{\mu 0} \mathcal{A}_{a}^{\mu} t^{a}$ (however the covariant derivative acting on the pions remains unchanged) and adding a phenomenological potential for the gluonic sector (discussed below). The Polyakov gauge is particularly convenient for calculations as the Wilson line is then a diagonal matrix, $L=e^{i\left(\lambda^{3} A_{3}+\lambda^{8} A_{8}\right) / 2 T}$. Utilizing this and the mean field solution for the effective potential the quark distribution functions are found to be transformed from the standard Fermi-Dirac distribution functions to

$$
\begin{aligned}
n_{F}^{+}(\Phi, \bar{\Phi} ; T, \mu) & =\frac{1+2 \bar{\Phi} e^{\beta\left(E_{q}-\mu\right)}+\Phi e^{2 \beta\left(E_{q}-\mu\right)}}{1+3 \bar{\Phi} e^{\beta\left(E_{q}-\mu\right)}+3 \Phi e^{2 \beta\left(E_{q}-\mu\right)}+e^{3 \beta\left(E_{q}-\mu\right)}}, \\
n_{F}^{-}(\Phi, \bar{\Phi} ; T, \mu) & =n_{F}^{+}(\bar{\Phi}, \Phi ; T,-\mu) .
\end{aligned}
$$

These are then substituted back into the renormalization group flow equation (2.6). This form is a particularly promising result, as in the confining limit ( $\Phi$ and $\bar{\Phi} \rightarrow 0$ ) we obtain a Fermi-Dirac-like distribution function for states of three quarks, however as $\Phi$ and $\bar{\Phi} \rightarrow 1$ the functions $n_{F}^{ \pm}$are equal to the standard Fermi-Dirac distribution functions, as they should be.

A number of forms for the gluonic potentials have been proposed and investigated at mean field level for the PNJL model [37] and the PQM model with $\mu=0$ [38]. In this work we will investigate three different gluon potentials. Since the Polyakov loop variable is the order parameter for the $Z(3)$ center symmetry of pure-glue QCD, a Ginzburg-Landau type potential should incorporate this. A polynomial expansion then leads to [39]

$$
\frac{U_{\text {poly }}}{T^{4}}=-\frac{b_{2}(T)}{2} \Phi \bar{\Phi}-\frac{b_{3}}{6}\left(\Phi^{3}+\bar{\Phi}^{3}\right)+\frac{b_{4}}{4}(\Phi \bar{\Phi})^{2},
$$

where the coefficients are

$$
\begin{aligned}
b_{2}(T) & =6.75-1.95\left(\frac{T_{0}}{T}\right)+2.624\left(\frac{T_{0}}{T}\right)^{2}-7.44\left(\frac{T_{0}}{T}\right)^{3}, \\
b_{3} & =0.75 \\
b_{4} & =7.5 .
\end{aligned}
$$

The coefficients $b_{2}(T), b_{3}$, and $b_{4}$ are chosen such that the Polyakov loop potential reproduces the equation of state and temperature dependence of $\Phi$ around the transition 
at $\mu=0$. The parameter $T_{0}$ is the transition temperature for pure-glue QCD lattice calculations [40].

In refs. [41, 42], another form for the Polyakov loop potential based on the SU(3) Haar measure was proposed:

$$
\frac{U_{\log }}{T^{4}}=-\frac{a(T)}{2} \Phi \bar{\Phi}+b(T) \ln \left[1-6 \bar{\Phi} \Phi+4\left(\Phi^{3}+\bar{\Phi}^{3}\right)-3(\bar{\Phi} \Phi)^{2}\right],
$$

where the coefficients are

$$
\begin{aligned}
& a(T)=3.51-2.47\left(\frac{T_{0}}{T}\right)+15.2\left(\frac{T_{0}}{T}\right)^{2}, \\
& b(T)=-1.75\left(\frac{T_{0}}{T}\right)^{3} .
\end{aligned}
$$

We note that the logarithmic term ensures that the magnitude of $\Phi$ and $\bar{\Phi}$ is constrained to be in the region between -1 and 1, i.e. the possible attainable values for the normalized trace of an element of the SU(3). Finally, Fukushima proposed a Polyakov loop potential in [43]

$$
\frac{U_{\mathrm{Fuku}}}{T^{4}}=-\frac{b}{T^{3}}\left(54 e^{-a T_{0} / T} \Phi \bar{\Phi}+\ln \left[1-6 \Phi \bar{\Phi}+4\left(\Phi^{3}+\bar{\Phi}^{3}\right)-3(\Phi \bar{\Phi})^{2}\right]\right),
$$

where the constants are $a=664 / 270$ and $b=(196.2 \mathrm{MeV})^{3}$ and we have added dependence upon the transition temperature, $T_{0}$.

A problem with all the Polyakov loop potentials proposed is that they are independent of the number of flavors and of the baryon chemical potential. However, we know that, for example, the transition temperature for the deconfinement transition is a function of $N_{f}$. In other words, one ought to incorporate the back-reaction from the fermions to the gluonic sector. In ref. [44], the authors use perturbative arguments to estimate the effects of the number of flavors and the baryon chemical potential on the transition temperature $T_{0}$. The functional form of $T_{0}$ is [45]

$$
T_{0}=T_{\tau} e^{-1 /\left(\alpha_{0} b\left(N_{f}, \mu\right)\right)},
$$

where

$$
b\left(N_{f}, \mu\right)=\frac{1}{6 \pi}\left(11 N_{c}-2 N_{f}\right)-\frac{16}{\pi} N_{f} \frac{\mu^{2}}{\left(\hat{\gamma} T_{\tau}\right)^{2}},
$$

and $T_{\tau}=1.77 \mathrm{GeV}, \alpha_{0}=0.304$. $\hat{\gamma}$ controls the curvature of $T_{0}$ as a function of $\mu$, and again following [45] we experiment with a range of values to study the effects. This is further discussed in the following section.

Let us finally make a few remarks about the complexity of the effective action. In the mean-field approximation [47-50] of the PNJL and PQM models at finite $\mu_{B}$, the effective action is complex if one considers it a function of the complex variables $\Phi$ and $\bar{\Phi}$. If one ignores the imaginary part of the effective potential, the effective potential becomes a real function of real variables. One can then find a minimum of the effective potential in the usual way. However, in this approach, one obtains $\Phi=\bar{\Phi}$, which is in disagreement with lattice results. In our calculations, we restrict $\Phi$ and $\bar{\Phi}$ to be real and we find $\Phi \neq \bar{\Phi}$, thus avoiding the problem. 


\section{Numerical implementation and the glue potential}

To find the equilibrium state values of the order parameters $\phi, \Phi$ and $\bar{\Phi}$ we numerically solve the flow equation (2.6) with the boundary condition specified by the tree level potential, eq. (2.4), on a grid in $\phi$ - $\Phi$ - $\bar{\Phi}$-space with $\phi \in[0,126] \mathrm{MeV}$ and $\Phi, \bar{\Phi} \in[0,1](\Phi$ and $\bar{\Phi}$ are real, as discussed section 3). Doing this at various values of $T, B$ and $\mu$ gives us $U_{k=0}(\phi, \Phi, \bar{\Phi} ; T, B, \mu)$, which we construct as a dimensionless quantity. In the derivation of the flow equation we have used $O(4)$ symmetry, thus for the boundary condition of the flow we set $h=0$, then when minimising with respect to $\phi$ we minimise $U_{k=0}-h \phi$. The resulting surface, $U_{k=0}(\Phi, \bar{\Phi})$ is very smooth thus we use interpolation to save computation time. Additional runs at intermediate values show that errors due to the interpolation are on the order of $0.1 \%$. Before we minimise with respect to the deconfinement order parameters we must add the gluonic potential. Thus $\Phi$ and $\bar{\Phi}$ are obtained from the minimisation of $U_{k=0}(\Phi, \bar{\Phi})+U_{\text {glue }}(\Phi, \bar{\Phi}) / \Lambda^{4}$, where 'glue' stands for one of 'poly', 'log' or 'Fuku' as given in section 3 .

We use the following (dimensionless) bare parameters: $m_{\Lambda}^{2}=0.075, \lambda_{\Lambda}=9.2, g=$ 3.2258 and $h=0.0146$ and we have $\Lambda=500 \mathrm{MeV}$ which give constituent quark masses of $300 \mathrm{MeV}$, a sigma mass of $\sim 478 \mathrm{MeV}$ and pion masses of $\sim 140 \mathrm{MeV}$, that is, our results are calculated at the physical point. Changing the energy of the ultraviolet cutoff from 500 to $800 \mathrm{MeV}$, gives an increase of approximately $3 \%$ to the chiral phase transition at low $\mu$, and approximately $10 \%$ at low $T$. Additional details about the implementation at $\Phi=\bar{\Phi}=1$ can be found in [17].

As the results presented here are calculated at the physical point all of the phase transitions are crossover 'transitions' and thus all critical temperatures are pseudo-critical temperatures. We must therefore define how we can calculate these transitions. Since we have discretized the variables in the computation of the effective potential, calculating the inflection point directly from the output data is very inaccurate. Thus one way to define the transition temperature is to fit the data points for the order parameter in question with a function and then define the transition temperature, $T_{\mathrm{x}}$, as the inflection point of the fitted curve. For the chiral transition we use this method, with the fit based on $\arctan (x)$. However, using this method for the deconfinement transition we run into problems as the functional form of the underlying curve changes with changing $\mu$ (see the left panel of figure 3). An alternative way of defining this transition is when the order parameter, $\Phi(T)$, is equal to $\frac{1}{2}$, this we define as $T_{\Phi / 2}$. To find this point we interpolate with third-order polynomial interpolation. Figure 1 illustrates this for $\mu=0$. The left panel shows the data points (crosses) for $\phi$ as a function of $T$. The open circle indicates the inflection point of the fitted curve, i.e. $T_{\phi}$, while the cross indicates the temperature when the normalized chiral order parameter satisfies $\phi / \phi(T=0)=\frac{1}{2}$, we denote this $T_{\phi / 2}$. The right panel shows the same, but now for the deconfinement order parameter $\Phi$ and the green curve is now the interpolation used to determine $T_{\Phi / 2}$.

Following ref. [44], we introduced an $N_{f}$ and $\mu_{B}$-dependent transition temperature $T_{0}$ via eq. (3.13). In figure 2 , we show the effects of varying the parameter $\hat{\gamma}$ in eq. (3.14) on the deconfinement transition in the $\mu-T$ plane for zero magnetic field and utilizing the 

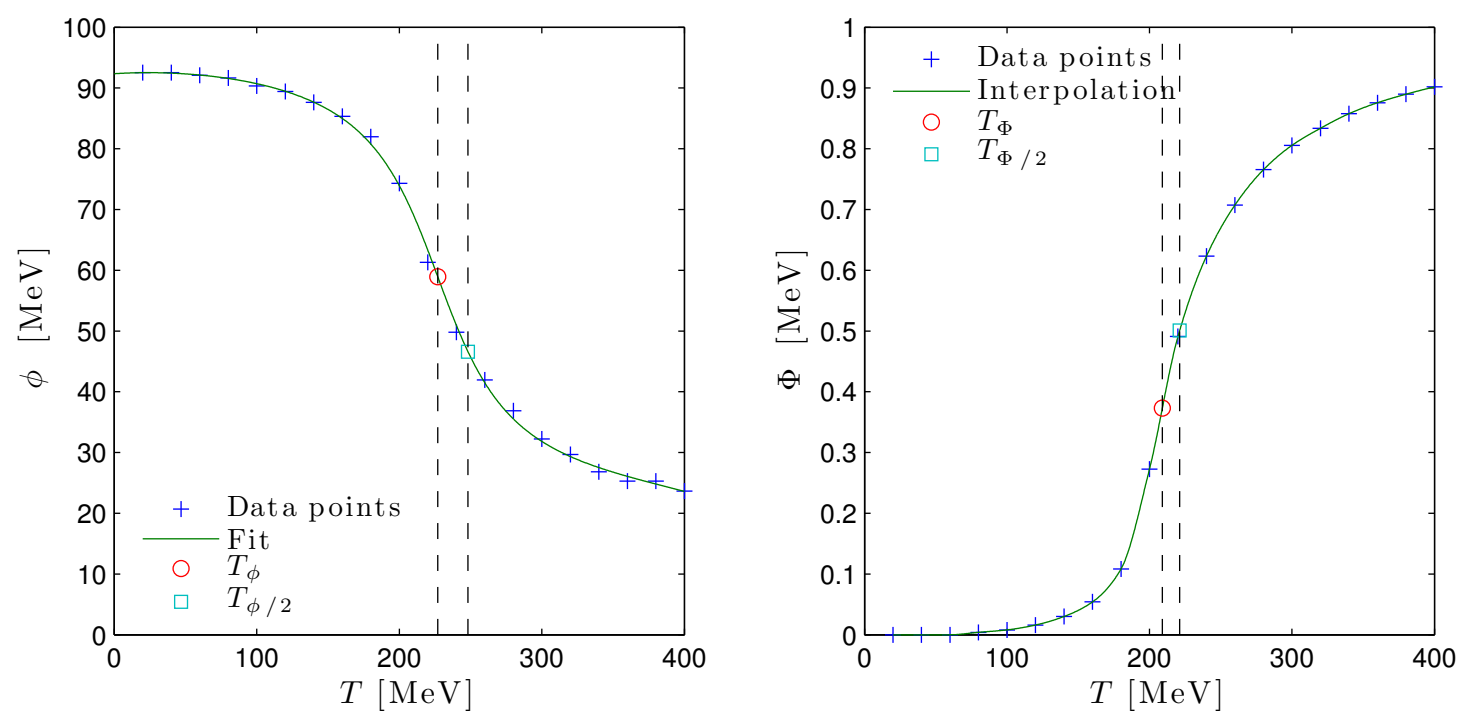

Figure 1. Methods used to determine the transition temperatures for the chiral transition (left) and deconfinement transition (right). Both plots are for $\mu=0$. See main text for details.

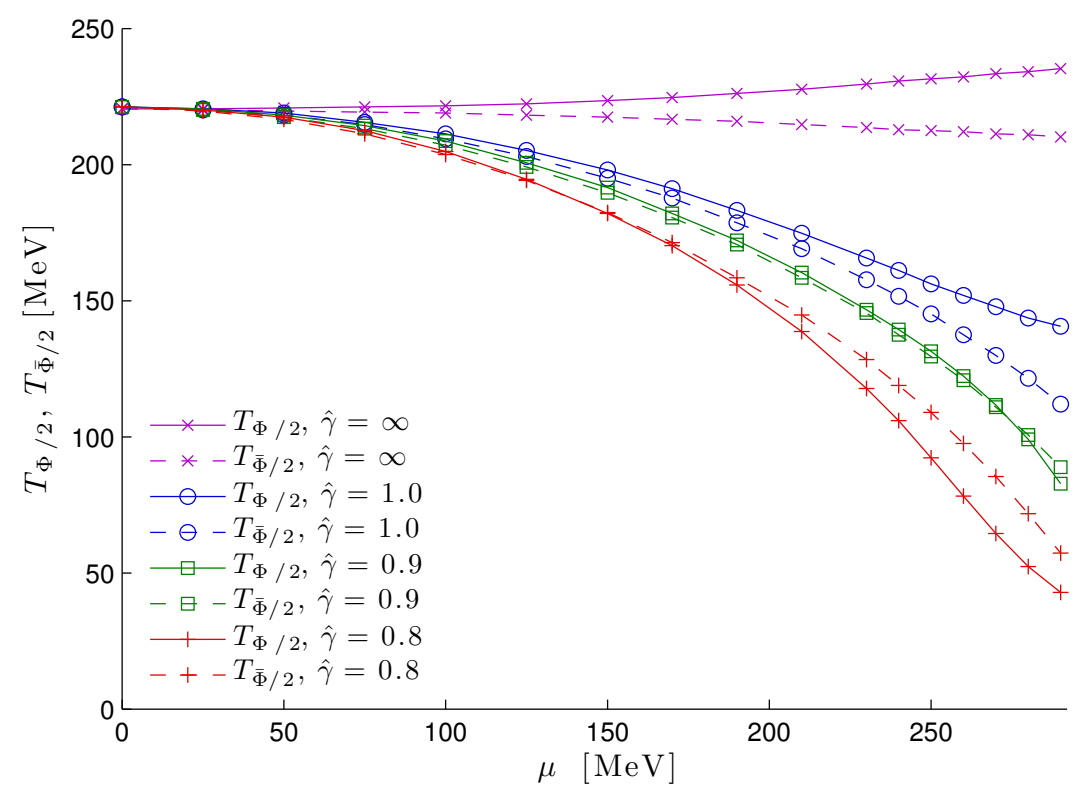

Figure 2. Phase diagram for the deconfinement transition with $B=0$ and various values of the parameter $\hat{\gamma}$. See main text for details.

polynomial gluonic potential, eq. (3.5). The solid lines show $T_{\Phi / 2}$ while the dashed lines show $T_{\bar{\Phi} / 2}$ for the same values of $\hat{\gamma}$. We note that both $\Phi$ and $\bar{\Phi}$ are real and coincide for $\mu=0$ but differ at non-zero $\mu$. Furthermore, for a $\mu_{B}$-independent $T_{0}(=208 \mathrm{MeV})$ the transition temperature is almost independent of the baryon chemical potential $\mu$ (magenta lines). The red, green, and blue lines show the results for $\hat{\gamma}=0.8,0.9$, and 1.0, respectively. The bending of the curves decreases as a function of $\hat{\gamma}$ which is reasonable since this 

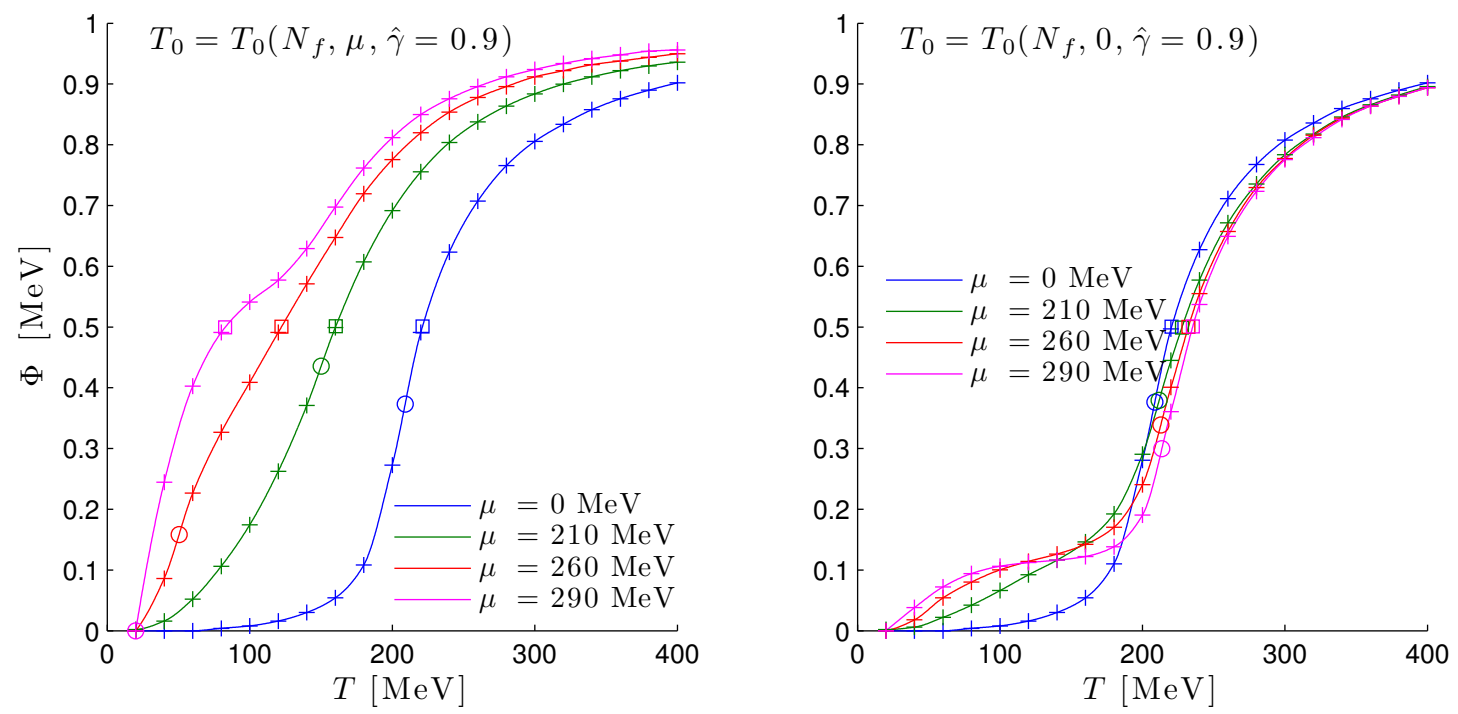

Figure 3. Order parameter $\Phi$ as a function of $T$ for various values of the chemical potential $\mu$ with (left) and without (right) a $\mu$ dependent gluonic transition temperature, $T_{0} .+\mathrm{s}$ are the data points, lines are the interpolations thereof, $\square$ s give $T_{\Phi / 2}$ and $\circ \mathrm{s}$ approximate $T_{\Phi}$.

parameter enters in the denominator of the parametrization $(3.14)$ of $b\left(N_{f}, \mu\right)$. At $\hat{\gamma} \approx 0.9$ we find the strongest $\mu$ dependence which still preserves $T_{\Phi / 2} \geq T_{\bar{\Phi} / 2}$ thus in the remaining figures in this paper (figures 3-8) we use $\hat{\gamma}=0.9$. Finally, we remark that the qualitative behavior is the same for finite magnetic field $B$. We will present more results for various $B$-fields in the next section.

In figure 3, we show the order parameter $\Phi(T)$ as a function of $T$ for $\mu=0$ (blue), $\mu=210$ (green), $\mu=260$ (red), and $\mu=290$ (magenta) with and without a $\mu$ dependent gluonic potential. In the left panel, the results are for $T_{0}=T_{0}\left(N_{f}, \mu\right)$, while in the right panel $T_{0}=T_{0}\left(N_{f}, 0\right)$ i.e. independence from $\mu$. Comparing the two panels we see the result shown in figure 2 , that only with a $\mu$ dependent transition temperature $T_{0}$ do we obtain significant change in the deconfinement order parameter when varying $\mu$. Additionally we see in the right panel that at high $\mu$ (magenta in particular) there is an initial increase in $\Phi$ around $T=50 \mathrm{MeV}$, which comes from the mesonic and fermionic potential, $U_{k=0}$, and then around $208 \mathrm{MeV}$ there is the typical increase, driven largely by the gluonic potential, $U_{\text {glue }}$. We then see in the left panel, with a $\mu$ dependent $T_{0}$, that the effect of $U_{\text {glue }}$ mirrors that of $U_{k=0}$ and the deconfinement transition thus decreases with increasing $\mu$.

Figure 3 also illustrates the aforementioned difficulties in defining the deconfinement transition at large $\mu$. It is seen that $T_{\Phi / 2} \sim T_{\Phi}$ at low $\mu$, but for $\mu \gtrsim 230 \mathrm{MeV}$ this is no longer true. In addition to this, the numerics become more time consuming at low $T$, thus for values of $T \gtrsim 30 \mathrm{MeV}$ our results only approximate the behavior of the model. For these reasons we have only calculated the phase diagram up to $\mu=290 \mathrm{MeV}{ }^{4}$

\footnotetext{
${ }^{4}$ We have also observed the splitting of the chiral transition reported in [46] without the Polyakov loop, but have not resolved that region in detail with the Polyakov loop.
} 


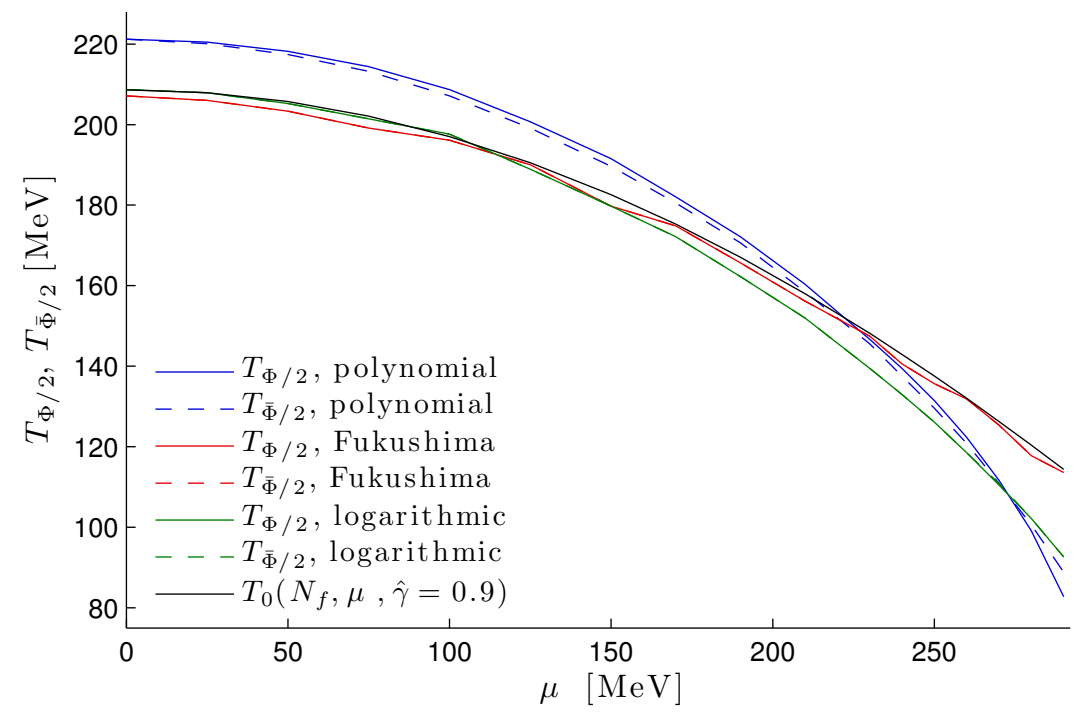

Figure 4. Phase diagram for the deconfinement transition for different glue potentials and $B=0$. Also shown is the transition temperature $T_{0}=T_{0}\left(N_{f}, \mu, \hat{\gamma}=0.9\right)$ for pure glue for comparison.

In figure 4, we show the phase diagram for the deconfinement transition with the three different glue potentials introduced in section 3 at $B=0$. The blue lines are the polynomial potential (3.5), the red lines are the logarithmic potential (3.9), and the green lines are the Fukushima potential (3.12). The black line shows the transition temperature $T_{0}=T_{0}\left(N_{f}, \mu, \hat{\gamma}=0.9\right)$ for pure glue for comparison. We note that the black curve is almost the same as the curve for the Fukushima potential (red), implying that the coupling to the quarks has almost no influence on the deconfinement transition.

As was observed in [41] we find with the logarithmic potential that $\Phi=\bar{\Phi}$ for all values of $\mu$, we also find this to be true with the Fukushima potential. We also find with the Fukushima potential, and to a lesser degree with the logarithmic potential, that the deconfinement transition temperature is dominated by the gluonic potential. This was also backed up by direct investigation of the $\Phi$ and $\bar{\Phi}$ as functions of $T$.

In figure 5 , we show the phase diagram for the chiral transition using the different gluonic potentials. We also show the phase diagram for the quark-meson model without the Polyakov loop, i.e. for $\Phi=1$. The lines show that the particular form of the gluonic potential is not as influential as we saw in the case of the deconfinement transition. At zero $\mu$ and $B, T_{\phi}$ decreases by $2 \%$ and $3 \%$ for the logarithmic and Fukushima potentials respectively. Only with $\mu \gtrsim 260 \mathrm{MeV}$ do we see a significantly larger deviation than this.

\section{$5 \quad$ Results at finite magnetic field}

In this section, we will present our main results and discuss them in some detail. In figure 6 , we show the phase diagram for the chiral and the deconfinement transitions for $B=0$ (blue lines) and for $|q B|=5.3 m_{\pi}^{2}$. The results are obtained using the polynomial glue potential (3.5). We will discuss the results in detail in connection with figure 7 , 


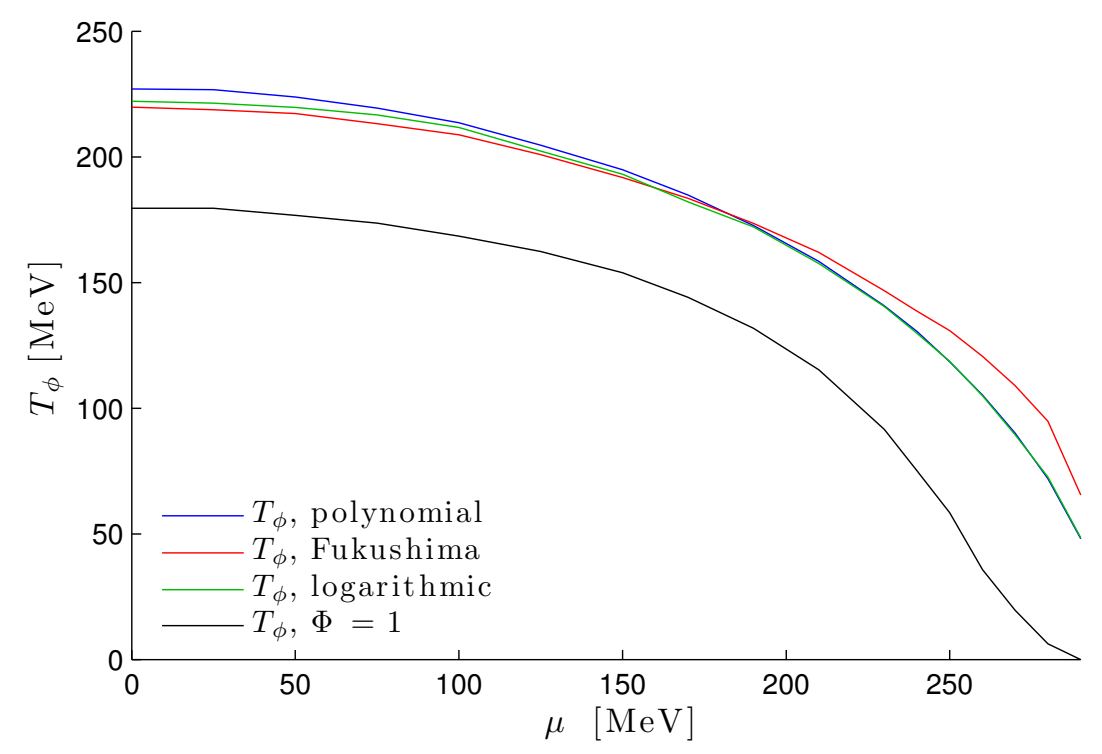

Figure 5. Phase diagram for the chiral transition for $B=0$ with different glue potentials however the same gluoinic transition temperature $T_{0}=T_{0}\left(N_{f}, \mu, \hat{\gamma}=0.9\right)$. Also shown is the transition temperature for $\Phi=1$, i.e. for the quark-meson model without the Polyakov loop.

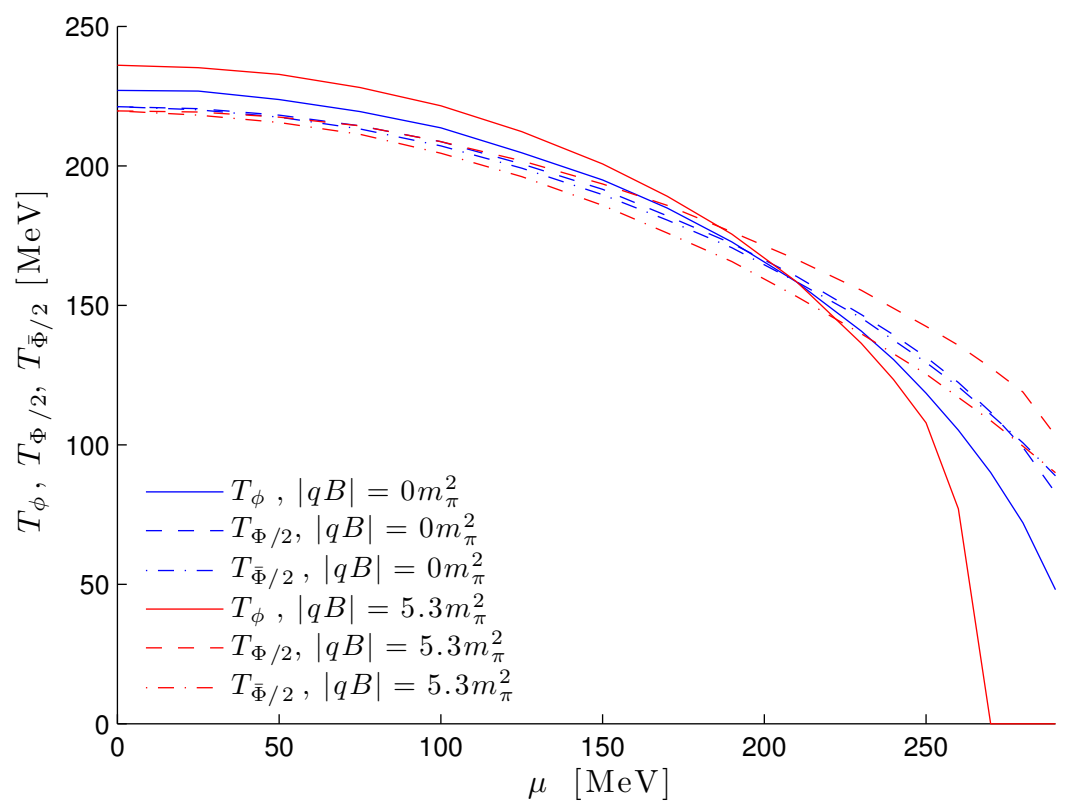

Figure 6. Phase diagram for the deconfinement and chiral transitions for $B=0$ and the largest magnetic field, $|q B|=5.3 \mathrm{~m}_{\pi}^{2}$ with the Polynomial potential.

where we show the chiral and deconfinement transition temperatures as a function of $B$ for different values of $\mu$.

In figure 7 , we show the transition temperatures for the chiral and deconfinement transitions as functions of $B$ for different values of $\mu$. The solid blue lines indicate the 

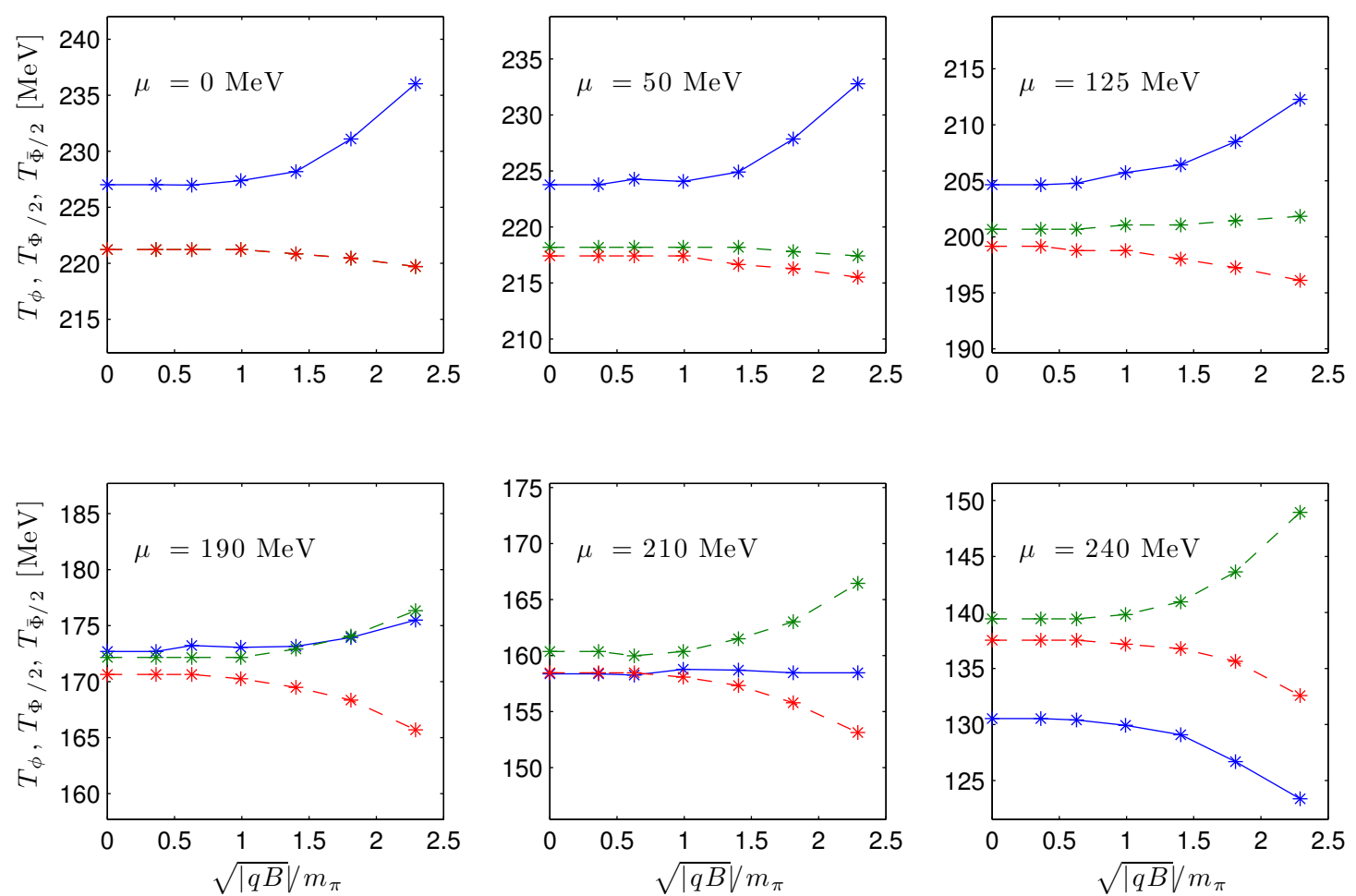

Figure 7. Transition temperatures for the chiral and deconfinement transitions as functions of $B$ for different values of $\mu$. Solid blue lines denote $T_{\phi}$ while dashed lines correspond to the deconfinement transition with green giving $T_{\Phi / 2}$, red giving $T_{\bar{\Phi} / 2}$.

chiral transition, $T_{\phi}$, while the dashed green lines are $T_{\Phi / 2}$ and the dashed red lines are $T_{\bar{\Phi} / 2}$. In the left upper panel, $\mu=0$ and $\Phi=\frac{1}{2}$ and $\bar{\Phi}=\frac{1}{2}$ coincide for all $B$. We note that the transition temperature for the chiral transition is increasing for values of $\mu$ up to approximately $\mu=210 \mathrm{MeV}$ where it is flat (lower middle panel). For larger chemical potentials, the transition temperature for chiral transition is a decreasing function. This shows the magnetic catalysis for small $\mu$ and inverse catalysis for large $\mu$ which we discuss below. For nonzero $\mu$ we see that the splitting between $\Phi$ and $\bar{\Phi}$ increases with $\mu$ and also with the strength of the magnetic field $B$. For small values of $\mu, T_{\Phi / 2}$ and $T_{\bar{\Phi} / 2}$ are almost independent of $B$, while for large values, $T_{\Phi / 2}$ increases with increasing $B$ while $T_{\bar{\Phi} / 2}$ decreases with $B$. This behavior indicates that the relative importance of the fermionic and mesonic fields also increases with larger $B$ and $\mu$ although we have not identified a mechanism behind this behavior.

In figure 8 , we show the phase diagram for the chiral phase transition for different values of the magnetic field $B$ with coupling to the Polyakov loop variable (solid lines) and without (dashed lines). Inset shows the transition temperature as a function of $B$ for vanishing $\mu$ in the two cases. We first notice that the critical temperature increases with the magnetic field for small values of the chemical potential $\mu$. The basic mechanism is that of magnetic catalysis [51-53], namely that the chiral condensate increases as a function of the magnetic field. It is interesting to note that the increase of the transition temperature 


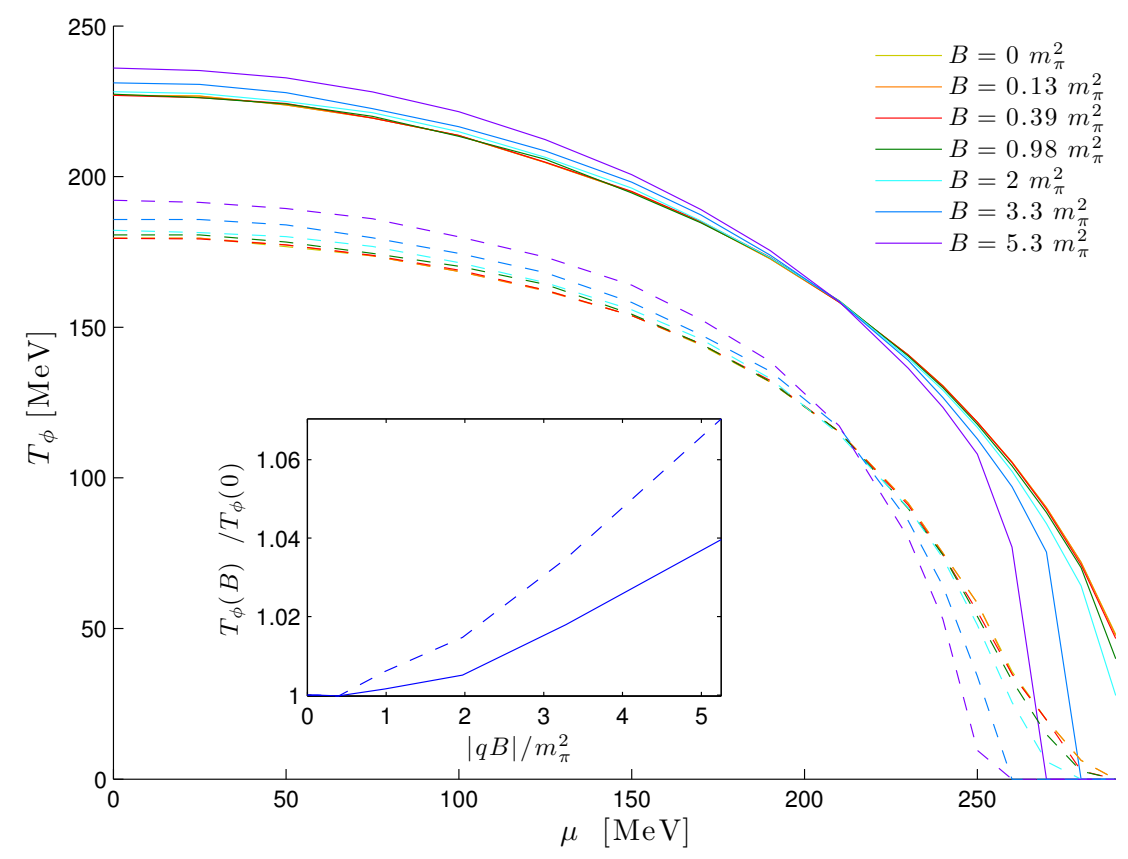

Figure 8. Phase diagram for the chiral transition for different values of the magnetic field $B$ with (solid lines) and without (dashed lines) the Polyakov loop using the polynomial potential. Inset shows the critical temperature as a function of $B$ for $\mu=0$ with (solid lines) and without (dashed lines) the Polyakov loop.

as a function of $B$ is smaller when we couple the chiral sector to the gluonic sector. For large values of the chemical potential $\mu$, the critical temperature is a decreasing function of the magnetic field. This is inverse catalysis $[54,55]$. We also find that the transition temperature is increased signficantly for all values of $\mu$ with the addition of the Polyakov loop. Below $\mu \sim 200 \mathrm{MeV} T_{\phi}$ increases by approximately $25 \%$ and above this density we find greater increases in $T_{\phi}$. The Polyakov loop acts to suppress the finite temperature, fermionic contribution to the effective potential at all temperatures, although particularly at low temperatures. Thus we expect some increase in $T_{\phi}$ but its magnitude is of interest as it shows that the confining dynamics does play an important role in the chiral transition within this model. In this region we find $T_{\phi}$, Fuku $-T_{\phi, \log / \text { poly }} \approx 20 \mathrm{MeV}$. The relative increase in magnetic field is more greatly affected by the choice of potential, with the relative increase in $T_{\phi}$ being approximately $20 \%$ less with the logarithmic and Fukushima potentials as opposed to the polynomial potential shown in figure 8 .

Very recently, the existence of a new critical point associated with the deconfinement transition of strongly interacting matter at finite $T$ and $B$, but vanishing $\mu$ has been suggested [56]. The basic idea is that quarks effectively decouple in the presence of very large magnetic fields due to their increasing mass as a function of $B$. In this case, one should be able to describe the system with an effective theory of pure gluondynamics. Although this effective theory is anisotropic, it is likely that it has a first-order transition just like isotropic pure-glue QCD. Since QCD with physical quark masses exhibit a crossover and 
not a first-order transition, there ought to be a critical point in the $T-B$ plane, where the line of first-order transition ends. However we find no evidence within the range of magnetic fields we examine of a transformation from the observed cross-over transition to a first order transition for the deconfinement order parameter.

\section{Summary and outlook}

In this work we have used the functional renormalization group to calculate the phase diagram with respect to the chiral and deconfinement transitions for the Polyakov loop extended quark-meson model. We first investigated the effects of the gluonic potential, showing that the deconfinement transision is quantitavely dependent upon the exact implementation, and in some cases even qualitatively dependent. Most noticeably $T_{\Phi / 2}-T_{\bar{\Phi} / 2}$ is only non-zero when using the polynomial potential (3.5). This potential was also the least dominating in that the fermionic and mesonic degrees of freedom had a much larger effect upon the deconfinement order parameters, $\Phi$ and $\bar{\Phi}$. However for all three potentials the gluonic potential dominated the dynamics. At high $\mu$ we see a double humped structure in these order parameters. This made the evaluation of $T_{\Phi / 2}$ and $T_{\bar{\Phi} / 2}$ difficult and we can not find a first order transition around $\mu \sim 300 \mathrm{MeV}$ (given by Herbst et al. [45]) although we saw indications of this.

We find magnetic catalysis at low $\mu$ in agreement with other model calculations, however we see a weakening of its effects with the addition of the Polyakov loop. At large $\mu$ the inverse magnetic catalysis found in the quark-meson model [17] is also found here. When using the polynomial potential we a find splitting of $T_{\Phi / 2}$ and $T_{\bar{\Phi} / 2}$ at non-zero $\mu$. This splitting increases with increasing magnetic field strength and quark chemical potential (other than for the very highest $\mu$ value). In addition $T_{\phi}$ increases significantly for all values of $\mu$ shows that the Polyakov loop plays an important role in the chiral transition. In contrast to the confinement transition, we found that the chiral transition is not sensitive to the choice of the gluon potential.

In the recent papers [6, 24], the authors suggest a resolution of the discrepancy between the model calculations and the lattice simulations. The chiral condensate can be written as

$$
\langle\bar{\psi} \psi\rangle=\frac{1}{\mathcal{Z}(B)} \int d \mathcal{U} e^{-S_{g}} \operatorname{det}(\not D(B)+m) \operatorname{Tr}(\not D(B)+m)^{-1},
$$

where the partition function is

$$
\mathcal{Z}(B)=\int d \mathcal{U} e^{-S_{g}} \operatorname{det}(\not D(B)+m)
$$

and $S_{g}$ is the pure-glue action. Thus there are two contributions to the chiral condensate, namely the operator itself (called valence contribution) and the change of typical gauge configurations sampled, coming from the determinant in eq. (6.1) (called sea contribution). At least for small magnetic fields one can disentangle these contributions by defining

$$
\begin{aligned}
\langle\bar{\psi} \psi\rangle^{\mathrm{val}} & =\frac{1}{\mathcal{Z}(0)} \int d \mathcal{U} e^{-S_{g}} \operatorname{det}(\not \mathcal{D}(0)+m) \operatorname{Tr}(\not D(B)+m)^{-1} \\
\langle\bar{\psi} \psi\rangle^{\text {sea }} & =\frac{1}{\mathcal{Z}(B)} \int d \mathcal{U} e^{-S_{g}} \operatorname{det}(\not D(B)+m) \operatorname{Tr}(\not D(0)+m)^{-1}
\end{aligned}
$$


At zero temperature, both contributions are positive leading to magnetic catalysis. At temperatures around the transition temperature, the valence condensate is still positive while the sea condensate is negative. Hence there is a competition between the two leading to a net inverse catalysis. The sea contribution can be viewed as a back reaction of the fermions on the gauge fields and this effect is not present in the model calculations as there are no dynamical gauge fields. If such a back reaction can be incorporated in the model calculations, one may be able to obtain agreement with the lattice simulations. One interesting attempt that was made recently, used a $B$-dependent parametrization of the transition temperature $T_{0}$ [28] in analogy with the flavor and $\mu_{B}$ dependence of $T_{0}$. Given the constraint that there is magnetic catalysis at zero temperature and that the chiral transition is a crossover, the authours found that the PQM model leads to thermal magnetic catalysis in the entire allowed parameter space. The calculations presented in [28] were in the mean-field approximation and whether the inclusion of bosonic fluctuations changes this picture is not known. In that case, we are still missing a key ingredient within these models and the disagreement with lattice remains a major challenge to model builders.

\section{Acknowledgments}

The authors would like to thank Kazuhiko Kamikado, Takuya Kanazawa, and Jonas R. Glesaaen for valuable discussions.

Open Access. This article is distributed under the terms of the Creative Commons Attribution License (CC-BY 4.0), which permits any use, distribution and reproduction in any medium, provided the original author(s) and source are credited.

\section{References}

[1] D.E. Kharzeev, L.D. McLerran and H.J. Warringa, The effects of topological charge change in heavy ion collisions: 'event by event P and CP-violation', Nucl. Phys. A 803 (2008) 227 [arXiv:0711.0950] [INSPIRE].

[2] V. Skokov, A.Y. Illarionov and V. Toneev, Estimate of the magnetic field strength in heavy-ion collisions, Int. J. Mod. Phys. A 24 (2009) 5925 [arXiv:0907.1396] [inSPIRE].

[3] A. Bzdak and V. Skokov, Event-by-event fluctuations of magnetic and electric fields in heavy ion collisions, Phys. Lett. B 710 (2012) 171 [arXiv:1111.1949] [INSPIRE].

[4] J.M. Lattimer and M. Prakash, Neutron star observations: prognosis for equation of state constraints, Phys. Rept. 442 (2007) 109 [astro-ph/0612440] [INSPIRE].

[5] D. Kharzeev, K. Landsteiner, A. Schmitt and H.-U. Yee eds., Strongly interacting matter in magnetic fields, Lect. Notes Phys. 871 (2013) 1 [INSPIRE].

[6] G.S. Bali et al., The QCD phase diagram for external magnetic fields, JHEP 02 (2012) 044 [arXiv:1111.4956] [INSPIRE].

[7] G.S. Bali et al., QCD quark condensate in external magnetic fields, Phys. Rev. D 86 (2012) 071502 [arXiv: 1206.4205] [INSPIRE]. 
[8] M. D'Elia, S. Mukherjee and F. Sanfilippo, QCD phase transition in a strong magnetic background, Phys. Rev. D 82 (2010) 051501 [arXiv: 1005.5365] [InSPIRE].

[9] M. D'Elia and F. Negro, Chiral properties of strong interactions in a magnetic background, Phys. Rev. D 83 (2011) 114028 [arXiv:1103.2080] [inSPIRE].

[10] A.J. Mizher, M.N. Chernodub and E.S. Fraga, Phase diagram of hot QCD in an external magnetic field: possible splitting of deconfinement and chiral transitions, Phys. Rev. D 82 (2010) 105016 [arXiv:1004.2712] [INSPIRE].

[11] S. Fayazbakhsh and N. Sadooghi, Phase diagram of hot magnetized two-flavor color superconducting quark matter, Phys. Rev. D 83 (2011) 025026 [arXiv:1009.6125] [InSPIRE].

[12] R. Gatto and M. Ruggieri, Dressed Polyakov loop and phase diagram of hot quark matter under magnetic field, Phys. Rev. D 82 (2010) 054027 [arXiv: 1007.0790] [InSPIRE].

[13] R. Gatto and M. Ruggieri, Deconfinement and chiral symmetry restoration in a strong magnetic background, Phys. Rev. D 83 (2011) 034016 [arXiv: 1012.1291] [INSPIRE].

[14] K. Kashiwa, Entanglement between chiral and deconfinement transitions under strong uniform magnetic background field, Phys. Rev. D 83 (2011) 117901 [arXiv:1104.5167] [INSPIRE].

[15] D.C. Duarte, R.L.S. Farias and R.O. Ramos, Optimized perturbation theory for charged scalar fields at finite temperature and in an external magnetic field, Phys. Rev. D 84 (2011) 083525 [arXiv: 1108.4428] [INSPIRE].

[16] V. Skokov, Phase diagram in an external magnetic field beyond a mean-field approximation, Phys. Rev. D 85 (2012) 034026 [arXiv:1112.5137] [INSPIRE].

[17] J.O. Andersen and A. Tranberg, The chiral transition in a magnetic background: finite density effects and the functional renormalization group, JHEP 08 (2012) 002 [arXiv:1204.3360] [INSPIRE].

[18] M. Ruggieri, M. Tachibana and V. Greco, Renormalized vs. nonrenormalized chiral transition in a magnetic background, JHEP 07 (2013) 165 [arXiv:1305.0137] [INSPIRE].

[19] K. Kamikado and T. Kanazawa, Chiral dynamics in a magnetic field from the functional renormalization group, JHEP 03 (2014) 009 [arXiv: 1312.3124] [INSPIRE].

[20] M. Ferreira, P. Costa and C. Providência, Deconfinement, chiral symmetry restoration and thermodynamics of $(2+1)$-flavor hot QCD matter in an external magnetic field, Phys. Rev. D 89 (2014) 036006 [arXiv:1312.6733] [INSPIRE].

[21] E.S. Fraga and L.F. Palhares, Deconfinement in the presence of a strong magnetic background: an exercise within the MIT bag model, Phys. Rev. D 86 (2012) 016008 [arXiv:1201.5881] [INSPIRE].

[22] K. Fukushima and Y. Hidaka, Magnetic catalysis versus magnetic inhibition, Phys. Rev. Lett. 110 (2013) 031601 [arXiv:1209.1319] [INSPIRE].

[23] T. Kojo and N. Su, The quark mass gap in a magnetic field, Phys. Lett. B 720 (2013) 192 [arXiv: 1211.7318] [INSPIRE].

[24] F. Bruckmann, G. Endrodi and T.G. Kovacs, Inverse magnetic catalysis and the Polyakov loop, JHEP 04 (2013) 112 [arXiv:1303.3972] [INSPIRE]. 
[25] J. Chao, P. Chu and M. Huang, Inverse magnetic catalysis induced by sphalerons, Phys. Rev. D 88 (2013) 054009 [arXiv: 1305.1100] [InSPIRE].

[26] M. Ferreira, P. Costa, D.P. Menezes, C. Providência and N. Scoccola, Deconfinement and chiral restoration within the $\mathrm{SU}(3)$ Polyakov-Nambu-Jona-Lasinio and entangled Polyakov-Nambu-Jona-Lasinio models in an external magnetic field, Phys. Rev. D 89 (2014) 016002 [arXiv: 1305.4751] [inSPIRE].

[27] V.D. Orlovsky and Y.A. Simonov, The quark-hadron thermodynamics in magnetic field, Phys. Rev. D 89 (2014) 054012 [arXiv:1311.1087] [INSPIRE].

[28] E.S. Fraga, B.W. Mintz and J. Schaffner-Bielich, A search for inverse magnetic catalysis in thermal quark-meson models, Phys. Lett. B 731 (2014) 154 [arXiv:1311.3964] [INSPIRE].

[29] C. Wetterich, Average action and the renormalization group equations, Nucl. Phys. B 352 (1991) 529 [inSPIRE].

[30] D. Ebert, K.G. Klimenko, M.A. Vdovichenko and A.S. Vshivtsev, Magnetic oscillations in dense cold quark matter with four fermion interactions, Phys. Rev. D 61 (2000) 025005 [hep-ph/9905253] [INSPIRE].

[31] S.S. Avancini, D.P. Menezes, M.B. Pinto and C. Providência, The QCD critical end point under strong magnetic fields, Phys. Rev. D 85 (2012) 091901 [arXiv:1202.5641] [INSPIRE].

[32] K. Fukushima, Chiral effective model with the Polyakov loop, Phys. Lett. B 591 (2004) 277 [hep-ph/0310121] [INSPIRE].

[33] E. Megias, E. Ruiz Arriola and L.L. Salcedo, Polyakov loop in chiral quark models at finite temperature, Phys. Rev. D 74 (2006) 065005 [hep-ph/0412308] [INSPIRE].

[34] D.F. Litim, Optimized renormalization group flows, Phys. Rev. D 64 (2001) 105007 [hep-th/0103195] [INSPIRE].

[35] N. Strodthoff, B.-J. Schaefer and L. von Smekal, Quark-meson-diquark model for two-color QCD, Phys. Rev. D 85 (2012) 074007 [arXiv: 1112.5401] [InSPIRE].

[36] K. Kamikado, N. Strodthoff, L. von Smekal and J. Wambach, Fluctuations in the quark-meson model for QCD with isospin chemical potential, Phys. Lett. B 718 (2013) 1044 [arXiv:1207.0400] [INSPIRE].

[37] O. Lourenço, M. Dutra, A. Delfino and M. Malheiro, Hadron-quark phase transition in a hadronic and Polyakov-Nambu-Jona-Lasinio models perspective,

Phys. Rev. D 84 (2011) 125034 [arXiv:1201.1239] [inSPIRE].

[38] B.-J. Schaefer, M. Wagner and J. Wambach, Thermodynamics of $(2+1)$-flavor QCD: confronting models with lattice studies, Phys. Rev. D 81 (2010) 074013 [arXiv:0910.5628] [INSPIRE].

[39] C. Ratti, M.A. Thaler and W. Weise, Phases of QCD: lattice thermodynamics and a field theoretical model, Phys. Rev. D 73 (2006) 014019 [hep-ph/0506234] [InSPIRE].

[40] F. Karsch, E. Laermann and A. Peikert, Quark mass and flavor dependence of the $Q C D$ phase transition, Nucl. Phys. B 605 (2001) 579 [hep-lat/0012023] [INSPIRE].

[41] S. Roessner, C. Ratti and W. Weise, Polyakov loop, diquarks and the two-flavour phase diagram, Phys. Rev. D 75 (2007) 034007 [hep-ph/0609281] [INSPIRE]. 
[42] C. Ratti, S. Roessner, M.A. Thaler and W. Weise, Thermodynamics of the PNJL model, Eur. Phys. J. C 49 (2007) 213 [hep-ph/0609218] [inSPIRE].

[43] K. Fukushima, Phase diagrams in the three-flavor Nambu-Jona-Lasinio model with the Polyakov loop, Phys. Rev. D 77 (2008) 114028 [Erratum ibid. D 78 (2008) 039902] [arXiv:0803.3318] [INSPIRE].

[44] B.-J. Schaefer, J.M. Pawlowski and J. Wambach, The phase structure of the Polyakov-quark-meson model, Phys. Rev. D 76 (2007) 074023 [arXiv:0704.3234] [INSPIRE].

[45] T.K. Herbst, J.M. Pawlowski and B.-J. Schaefer, The phase structure of the Polyakov-quark-meson model beyond mean field, Phys. Lett. B 696 (2011) 58 [arXiv: 1008.0081] [INSPIRE].

[46] T.K. Herbst, J.M. Pawlowski and B.-J. Schaefer, Phase structure and thermodynamics of QCD, Phys. Rev. D 88 (2013) 014007 [arXiv: 1302.1426] [InSPIRE].

[47] A. Dumitru, R.D. Pisarski and D. Zschiesche, Dense quarks, and the fermion sign problem, in a $\mathrm{SU}(N)$ matrix model, Phys. Rev. D 72 (2005) 065008 [hep-ph/0505256] [INSPIRE].

[48] K. Fukushima and Y. Hidaka, A model study of the sign problem in the mean-field approximation, Phys. Rev. D 75 (2007) 036002 [hep-ph/0610323] [INSPIRE].

[49] S. Roessner, T. Hell, C. Ratti and W. Weise, The chiral and deconfinement crossover transitions: PNJL model beyond mean field, Nucl. Phys. A 814 (2008) 118 [arXiv: 0712.3152] [INSPIRE].

[50] B.W. Mintz, R. Stiele, R.O. Ramos and J. Schaffner-Bielich, Phase diagram and surface tension in the three-flavor Polyakov-quark-meson model, Phys. Rev. D 87 (2013) 036004 [arXiv: 1212.1184] [INSPIRE].

[51] K.G. Klimenko, Three-dimensional Gross-Neveu model at nonzero temperature and in an external magnetic field, Z. Phys. C 54 (1992) 323 [INSPIRE].

[52] V.P. Gusynin, V.A. Miransky and I.A. Shovkovy, Catalysis of dynamical flavor symmetry breaking by a magnetic field in (2+1)-dimensions, Phys. Rev. Lett. 73 (1994) 3499 [Erratum ibid. 76 (1996) 1005] [hep-ph/9405262] [INSPIRE].

[53] I.A. Shovkovy, Magnetic catalysis: a review, Lect. Notes Phys. 871 (2013) 13 [arXiv:1207.5081] [INSPIRE].

[54] T. Inagaki, D. Kimura and T. Murata, Four fermion interaction model in a constant magnetic field at finite temperature and chemical potential,

Prog. Theor. Phys. 111 (2004) 371 [hep-ph/0312005] [INSPIRE].

[55] F. Preis, A. Rebhan and A. Schmitt, Inverse magnetic catalysis in dense holographic matter, JHEP 03 (2011) 033 [arXiv: 1012.4785] [INSPIRE].

[56] T.D. Cohen and N. Yamamoto, New critical point for QCD in a magnetic field, Phys. Rev. D 89 (2014) 054029 [arXiv:1310.2234] [InSPIRE]. 